\title{
Cosmological constraints on matter density perturba- tions amplitude, neutrino mass and number of relativistic species
}

\author{
Rodion Burenin ${ }^{1, *}$ \\ ${ }^{1}$ Space Research Institute of RAS (IKI), Moscow, Russia
}

\begin{abstract}
It is shown that Planck CMB temperature anisotropy data at high multipoles, $\ell>1000$, produce the measurement of matter density perturbations amplitude that contradict to all other constraints obtained both from remaining Planck CMB anisotropy data and from other cosmological data, at about 3.7 $\sigma$ significance level. With the exception of Planck CMB temperature anisotropy data at high multipoles, all other measurements of density perturbation amplitude are in good agreement between each other and give the following measurements of linear density perturbation amplitude: $\sigma_{8}=0.792 \pm 0.006$, mean density of the Universe: $\Omega_{m}=0.287 \pm 0.007$, and Hubble constant: $H_{0}=69.4 \pm 0.6 \mathrm{~km} \mathrm{~s}^{-1} \mathrm{Mpc}^{-1}$. Therefore, in this case the tensions in $H_{0}$ constraints between Planck+BAO data and direct $H_{0}$ measurements are weaken, and the tensions in $\sigma_{8}$ measurements between Planck CMB data and large scale structure data disappear completely. Taking in account the data on baryon acoustic oscillations and (or) direct measurements of the Hubble constant, one can obtain different constraints on sum of neutrino mass and number of relativistic species.
\end{abstract}

\section{Introduction}

The measurements of cosmic microwave background (CMB) temperature and polarization obtained in Planck all-sky survey [1,2], constitute one of the basic cosmological datasets, currently used to constrain parameters of cosmological model. However, it is known that there is a number of tensions between cosmological parameters constraints, which are obtained from different subsets of Planck survey data as well from the data of some other cosmological measurements [e.g., 2-6].

Here we compare various constraints on linear matter density perturbations amplitude, obtained using various observables and various cosmological data such as measurements of CMB anisotropy, galaxy cluster mass function, weak gravitation lensing, matter power spectrum and redshift space distortions. It is shown that the cosmological parameters constraints obtained using these heterogeneous data are in good agreement with each other, with the exception of the data on Planck CMB temperature anisotropy spectrum at high multipoles, $\ell>1000$. The cosmological parameters constraints, which can be obtained with no use of this part of Planck survey data, are studied in detail. More detailed description of these constraints and further discussion can be found in [7].

\footnotetext{
*e-mail: rodion@hea.iki.rssi.ru
} 

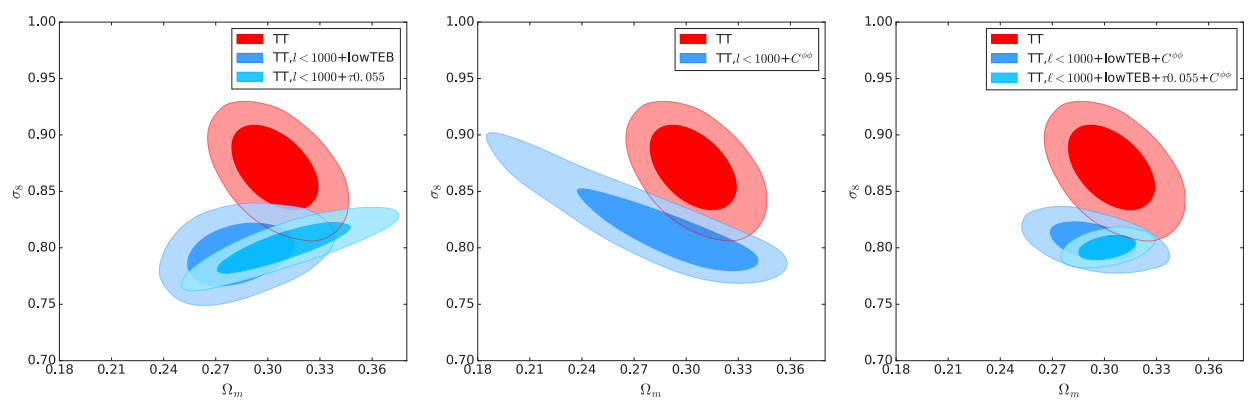

Figure 1. The constraints on mean matter density, $\Omega_{m}$, and linear density perturbations amplitude, $\sigma_{8}$, in $\Lambda C D M$ model, from Planck CMB temperature anisotropy spectrum (TT, red contours), and also from the data on CMB polarization at low multipoles (lowTEB, $\tau 0.055)$ and lensing potential $\left(C^{\phi \phi}\right)$, in combination with Planck CMB temperature anisotropy data at $\ell<1000$.

\section{Planck CMB anisotropy data}

CMB anisotropy data allow to measure the density perturbation amplitude in several ways. The constraints on, $\sigma_{8}$ - linear density perturbation amplitude at $8 \mathrm{Mpc}$ scale, obtained from Planck CMB temperature anisotropy power spectrum [2, 4], are shown in Fig. 1 with red contours. This constraint is obtained mainly from the measurement of the amplitude of CMB gravitation lensing on large scale structure, which is observed as smoothing of acoustic peaks at high multipoles, approximately at $\ell>1000$ [see, e.g., 7].

From the data on the CMB polarization at low multipoles one can obtain the constraint on reionization optical depth, $\tau$, which allow to constrain density perturbation amplitude using the measurement of the CMB temperature anisotropy amplitude itself. The constraints from CMB polarization data from Planck Low Frequency Instrument (LFI) [2, 8], and also from High Frequency Instrument (HFI) [9, 10] are shown in the left panel of Fig. 1. In order to do not mix these constrains with the constraints from CMB temperature anisotropy spectrum at high multipoles discussed above, the constraints from individual datasets are combined with Planck TT data at $\ell<1000$.

The density perturbations amplitude can also be constrained directly from the amplitude of observed lensing potential spectrum, $C^{\phi \phi}$, which can be recovered from four-point statistics (trispectrum) which was also done using Planck survey data [11]. The constrains on $\Omega_{m}$ and $\sigma_{8}$, obtained with these data are shown in the middle panel of Fig. 1. The $\Omega_{m}$ and $\sigma_{8}$ constraints obtained from the combined CMB polarization data at low multipoles and CMB lensing potential data are shown in the right panel of Fig. 1. These data, taken together, give the constraint $\sigma_{8}=0.800 \pm 0.007$, while Planck TT data give $\sigma_{8}=0.870 \pm 0.025$. Therefore, there is approximately $2.7 \sigma$ tension between these datasets.

These internal tensions in Planck CMB data were discussed in earlier works. The tensions between the parameters of $\triangle C D M$ model obtained from $C M B$ temperature anisotropy data at multipoles below and above $\ell=1000$ were discussed by [3]. These tensions were also discussed in Planck Collaboration papers $[2,4,6]$ where their significance was found to be not compelling enough. Recently [12] found that these tensions can be detected in Planck CMB temperature spectrum at about $2.4 \sigma$ significance in a model independent way. 

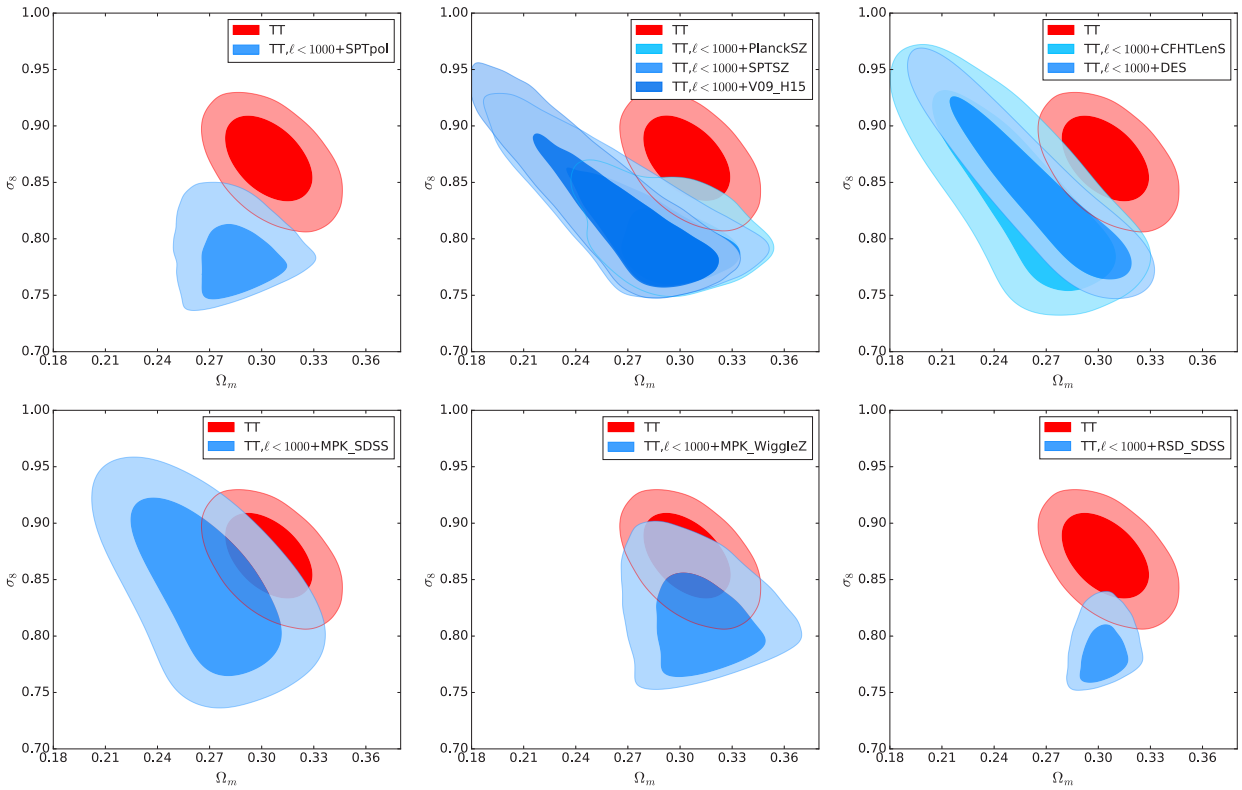

Figure 2. The constraints on mean matter density, $\Omega_{m}$, and linear density perturbations amplitude, $\sigma_{8}$, in $\Lambda C D M$ model from Planck survey CMB temperature anisotropy spectrum (TT, red contours), and also from the following data: upper row, left panel - South Pole Telescope CMB polarization anisotropy measurements (SPTpol), middle panel - galaxy cluster mass function measurements (PlanckSZ, SPTSZ, V09_H15), right panel — weak lensing of distant galaxies (CFHTLenS, DES), lower row, left panel - power spectrum of galaxies in SDSS 4-th data release (MPK_SDSS), middle panel — power spectrum of galaxies in WiggleZ survey (MPK_WiggleZ), right panel — redshift space distortions measured in SDSS 12-th data release $\left(R S D \_S D S S\right)$. All these data are combined with Planck CMB temperature anisotropy data at $\ell<1000$.
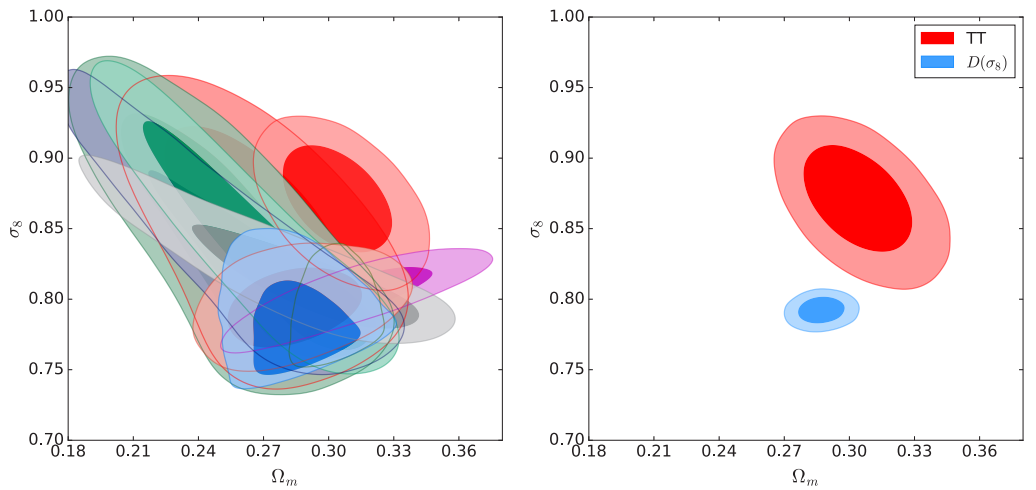

Figure 3. The constraints on mean matter density, $\Omega_{m}$, and linear density perturbations amplitude, $\sigma_{8}$, in $\Lambda C D M$ model from Planck survey CMB temperature anisotropy spectrum data (TT, red contours), and also from all other data presented in Fig. 2, taken together. 


\section{Other measurements of density perturbation amplitude}

\subsection{South Pole Telescope CMB polarization anisotropy data}

Gravitation lensing produce the smoothing of acoustic peaks not only in the spectrum of CMB temperature anisotropy, but also in CMB polarization spectrum. High sensitive measurements of CMB polarization anisotropy were obtained recently using the data of new SPTpol detector at South Pole Telescope [13]. The constraints on mean matter density $\Omega_{m}$, and linear density perturbations amplitude, $\sigma_{8}$, in $\Lambda C D M$ model, obtained with these data are presented in Fig. 2, upper row, left panel.

These data constrain $\sigma_{8}$ at notably lower values, as compared to Planck temperature anisotropy spectrum data (TT, red contours). This tension was discussed by SPTpol collaboration [13], who also note that it originates due to the SPTPol data at high multipoles, $\ell>1000$, while the data at lower multipoles, $\ell<1000$, are found to be in agreement with Planck survey data. We note, that SPTPol data both at low and high multipoles taken separately, produce only weak constraint on density perturbations amplitude. More accurate $\sigma_{8}$ measurement can be obtained only with SPTPol data at all multipoles, which are shown in Fig. 2 and are used below.

\subsection{Galaxy clusters}

The measurements of galaxy clusters mass function give one of the most sensitive methods to constrain matter density perturbations amplitude. These measurements were obtained in many works, based on cluster samples selected in X-rays [e.g., 14-16], optical [e.g., 17], and also using the observations of Sunyaev-Zeldovich effect [SZ, 18] in microwave band [e.g., 19, 20]. All these measurements produce approximately results, since their accuracy is limited by the uncertainty of cluster mass scale calibration.

Fig. 2, upper row, middle panel, shows the constraints on linear density perturbations amplitude, $\sigma_{8}$, obtained from the various galaxy cluster mass function datasets - the galaxy cluster data of Planck SZ survey [PlanckSZ, 20], South Pole Telescope [SPTSZ, 19], and also the data on clusters selected in X-rays from [14, 15], see also [21]. In the last case the constraints were corrected on the basis of recent cluster mass scale calibrations [22], and were taken in the form: $\sigma_{8}=0.821 \pm 0.027\left(\Omega_{m} / 0.25\right)^{0.4}$ [see details in 23]. These constraints can not be considered as completely independent ones, since very similar cluster mass scale calibrations are used. In our work we use only the last constraints based on X-ray selected galaxy clusters sample from $[14,15]$, taking in account new cluster mass scale calibrations — these data are referred to as V09_H15 below.

\subsection{Weak gravitational lensing}

The measurements of weak gravitational lensing of distant galaxies in deep optical surveys allow to measure inhomogeneity of matter distribution that light passes, which in turn allow to obtain almost direct measurement of matter density perturbation amplitude. The right panel in the upper row of Fig. 2 shows the $\sigma_{8}$ constraints from the data of Canada-France Hawaii Telescope Lensing Survey [CFHTLenS, 24], and also the constraints from the Dark Energy Survey first data release [DES, 25].

\subsection{Matter power spectrum and redshift space distortions}

Left panel in lower row of Fig. 2 shows the constraints on density perturbations amplitude from the data on power spectrum of galaxies in SDSS 4-th data release [MPK_SDSS, 26]. 
In lower row of Fig. 2 the constraints from the measurements of power spectrum of galaxies in WiggleZ survey [middle panel, MPK_WiggleZ, 27, 28], and also redshift space distortions measured in SDSS 12-th data release [right panel, RSD_SDSS, 29] are also shown. Note, that the constrains from two last datasets contain also the results of baryon acoustic oscillations measurements obtained from the same data.

\subsection{Combined constraints}

In the left panel of Fig. 3 all the constraints discussed above are shown. All these data are in good agreement with each other, with the exception of the data on Planck CMB temperature anisotropy spectrum at high multipoles, $\ell>1000$, which give significantly different $\sigma_{8}$ and $\Omega_{m}$ constraints. The combined constraints shown in the right panel of Fig. 3 are obtained as a combination of the following data:

- Planck survey CMB temperature anisotropy spectrum at multipoles $\ell<1000($ TT, $\ell<$ 1000);

- Planck LFI CMB polarization data at low multipoles, $\ell<30$ (lowTEB);

- Planck HFI CMB polarization data at low multipoles, $\ell<30$, used as a prior for reionization optical depth $\tau=0.055 \pm 0.009(\tau 0.055)$;

- Planck CMB gravitational lensing potential measurements $\left(C^{\phi \phi}\right)$;

- South Pole Telescope CMB polarization measurements (SPTpol);

- galaxy cluster mass function measurements from X-ray selected clusters (V09_H15);

- weak gravitational lensing of distant galaxies in Canada-France Hawaii Telescope Lensing Survey (CFHTLenS);

- weak gravitational lensing of distant galaxies in Dark Energy Survey, first data release $(D E S)$;

- luminous red galaxies power spectrum from Sloan Digital Sky Survey, data release 4 (MPK_SDSS).

The data on galaxies power spectrum in WiggleZ survey and the redshift space distortions data are not used here, because these data contain also the results of BAO measurements, which will be discussed below. In total, we use nine independent datasets, which are in good agreement among each other. Note, that almost all available data which provide direct constraints on on matter density perturbations amplitude are used, excluding the data on Planck CMB temperature anisotropy spectrum at high multipoles, $\ell>1000$. This combined dataset is referred as $D\left(\sigma_{8}\right)$ below.

The $\sigma_{8}$ and $\Omega_{m}$ constraints obtained with these data appears to be significantly different, as compared to the constraints from Planck CMB temperature anisotropy spectrum at high multipoles, $\ell>1000$ (see Fig. 3). The disagreement between these two datasets is significant at about $3.7 \sigma$ level. The observed significance corresponds to only one deviation of such amplitude in out of the order of 5000 realizations. In our case the number of independent trials can be estimated as the number of independent experiments, i.e. $\sim 10$, and therefore this disagreement should be considered as a significant one. The real reasons of this disagreement should be studied further. However, already now it is clear that the combination of these data should not be used currently to constrain cosmological parameters.

The $D\left(\sigma_{8}\right)$ combined dataset consist of many different measurements of density perturbation amplitude, which are found to be in good agreement between each other. Therefore, the cosmological parameters constraints based on this dataset should be reliable and 

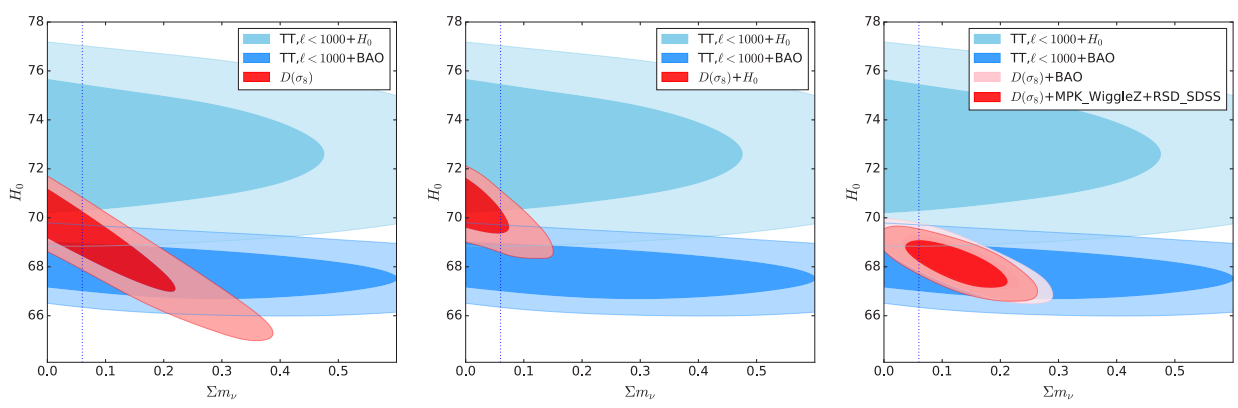

Figure 4. The constraints on sum of neutrino mass and Hubble constant from the BAO and direct $H_{0}$ data, and also from $D\left(\sigma_{8}\right)$ dataset in $\Lambda C D M+m_{v}$ model. Vertical dotted line show the value $\Sigma m_{v}=$ $0.06 \mathrm{eV}$, which approximately corresponds to the minimum sum of neutrino mass from the observations of neutrino oscillations.

they are studied in detail in [7]. These constraints give the following measurements of linear density perturbation amplitude: $\sigma_{8}=0.792 \pm 0.006$, mean density of the Universe: $\Omega_{m}=0.287 \pm 0.007$, and Hubble constant: $H_{0}=69.4 \pm 0.6 \mathrm{~km} \mathrm{~s}^{-1} \mathrm{Mpc}^{-1}$. The best estimates of $\sigma_{8}$ and $\Omega_{m}$ parameters are shifted to lower values, while the best estimate of Hubble constant, $H_{0}$, appears to be shifted high, as compared to Planck 2015 constraints. Note, that these constraints are in much better agreement with the pre-Planck constraints, which were obtained from the combination of various cosmological data [21,30], as compared to Planck 2015 constraints.

\section{The measurements of distances and expansion rate of Universe}

The data on baryon acoustic oscillations, type Ia supernovae and direct measurements of Hubble constant can not be used to obtain direct constraints on density perturbation amplitude. It can be shown that these data are in good agreement with both the data on Planck TT spectrum at high multipoles and with $D\left(\sigma_{8}\right)$ dataset [7]. There is only a weak, approximately $2 \sigma$, tension with the direct Hubble constant measurement. The constraints from the $D\left(\sigma_{8}\right)$ dataset are located approximately in between the constraints from BAO and direct measurement of Hubble constant.

\section{Constraints on sum of neutrino mass and number of relativistic species}

The constraints on sum of neutrino mass and Hubble constant from the $\mathrm{BAO}$ and direct $H_{0}$ data, and also from $D\left(\sigma_{8}\right)$ dataset in $\Lambda C D M+m_{v}$ model are shown in Fig. 4. Vertical dotted line show the value $\Sigma m_{v}=0.06 \mathrm{eV}$, which approximately corresponds to the minimum sum of neutrino mass from the observations of neutrino oscillations. One can see, that $H_{0}$ constraints from BAO and $H_{0}$ data do not depend on sum of neutrino mass, as expected. The $D\left(\sigma_{8}\right)$ dataset also give rather good constraint on $H_{0}$, but this constraint is strongly degenerate with the sum of neutrino mass. It can be shown that degeneracy is caused, eventually, by the suppression of density perturbation growth by massive neutrinos.

From Fig. 4 one can see that different combinations of datasets can give different $\Sigma m_{v}$ constraints. So, for example, $D\left(\sigma_{8}\right)$ taken separately produce the constraint: $\Sigma m_{v}<0.308 \mathrm{eV}$, while in combination with direct $H_{0}$ measurements this same dataset gives: $\Sigma m_{v}<0.117 \mathrm{eV}$. 

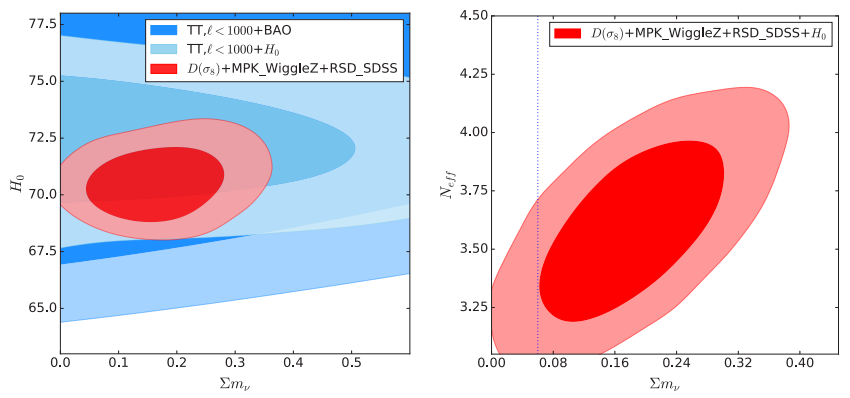

Figure 5. The constraints on sum of neutrino mass, $\Sigma m_{v}$, and number of relativistic species, $N_{\mathrm{eff}}$, in $\Lambda C D M+m_{v}+N_{\text {eff }}$ model, from the $D\left(\sigma_{8}\right)+M P K_{-}$WiggleZ $+R S D \_S D S S+H_{0}$ combined dataset.

Note, that in the last case the upper limit (at $95 \%$ confidence level) turns out to be close to the lower limit for sum of neutrino mass in the case of inverse mass hierachy, $\Sigma m_{v} \approx 0.1 \mathrm{eV}$ [e.g., 31].

In combination with the $\mathrm{BAO}$ data the $D\left(\sigma_{8}\right)$ dataset give the measurement of non-zero sum of neutrino mass, at approximately $2 \sigma$ significance: $\Sigma m_{v}=0.128 \pm 0.056 \mathrm{eV}$, the $95 \%$ upper limit in this case is $\Sigma m_{v}<0.233 \mathrm{eV}$. However, the measurement of Hubble constant based on BAO observations is also model dependent, since it is obtained using the sound horizon size as a standard ruler. Therefore, if one add the effective number of relativistic species, $N_{\text {eff }}$, as a free parameter to cosmological model, one could reconcile all the data discussed above. The $\Sigma m_{v}$ and $N_{\text {eff }}$ constraints in that model, using all the cosmological data discussed above $\left(D\left(\sigma_{8}\right)+M P K_{-}\right.$WiggleZ $\left.+R S D \_S D S S+H_{0}\right)$, are: $\Sigma m_{v}=0.189 \pm 0.080 \mathrm{eV}$ and $N_{\text {eff }}=3.61 \pm 0.25$. These constraints are shown in Fig. 5 .

Qualitatively, similar constraints were obtained earlier when the data on galaxy cluster mass function were combined with other cosmological data before the release of first Planck survey results [21, 30? ]. However, according to new constraints, allowed region of parameter space appears to be much smaller in size and shifted closer to the standard values of $\Lambda C D M$ model. The remaining deviations of $\Sigma m_{v}$ and $N_{\text {eff }}$ from $\Lambda C D M$ standard values are driven by the remaining tensions between the direct $H_{0}$ measurements and BAO observations.

\section{Discussion and conclusions}

We consider various measurements of density perturbations amplitude based on the CMB temperature and polarization anisotropy spectra, CMB lensing potential, galaxy cluster mass function, weak gravitational lensing of distant galaxies, matter power spectrum and redshift space distortions in spectroscopic surveys of galaxies. All these measurements were carried out in different experiments, are completely independent and have different systematic uncertainties.

It turns out that all these measurements are in good agreement with each other, with the exception of the data on Planck CMB temperature anisotropy spectrum at high multipoles, $\ell>1000$. This measurement contradict to all other constraints obtained both from remaining Planck CMB anisotropy data and from other cosmological data, at about $3.7 \sigma$ significance level. The true explanation of this disagreement should be identified in a separate study. However, already now it is clear that the combination of these data should not be used currently to constrain cosmological parameters. If Planck CMB temperature anisotropy spectrum data at high multipoles, $\ell>1000$, are not concidered, the tensions in $H_{0}$ constraints between 
Planck+BAO data and direct $H_{0}$ measurements are weaken, and the tensions in $\sigma_{8}$ measurements between Planck and large scale structure data disappear completely.

The combined $D\left(\sigma_{8}\right)$ dataset give the Hubble constant measurement, which is located approximately in between the constraints from the BAO and direct $H_{0}$ measurements. From the different combination of these data one can obtain different constraints on sum of neutrino mass. All these data can be reconciled completely, if one assume non-zero sum of neutrino mass and larger than standard number of relativistic species.

The work is supported by Russian Science Foundation grant 18-12-00520. This project was carried out using the computational resources of Spectrum-Roentgen-Gamma project data archive at IKI and also the resources of MVS-10P computer cluster at Joint Supercomputer Center of the Russian Academy of Sciences (JSCC RAS).

\section{References}

[1] Planck Collaboration, Planck 2015 Results I, A\&A, 594, A1 (2016)

[2] Planck Collaboration, Planck 2015 Results XIII, A\&A, 594, A13 (2016)

[3] Addison G.E., Huang Y., Watts D.J., et al., ApJ, 818, 132 (2016)

[4] Planck Collaboration, Planck 2015 Results XI, A\&A, 594, A11 (2016)

[5] Couchot F., Henrot-Versillé, S., Perdereau O., et al., A\&A, 597, A126 (2017)

[6] Planck Collaboration LI, A\&A, 607, A95 (2017)

[7] Burenin R.A., Astronomy Letters, in press; arXiv:1806.03261 (2018)

[8] Planck Collaboration, Planck 2015 Results VI, A\&A, 594, A6 (2016)

[9] Planck Collaboration XLVI, A\&A, 596, A107 (2016)

[10] Planck Collaboration XLVII, A\&A, 596, A108 (2016)

[11] Planck Collaboration, Planck 2015 Results XV, A\&A, 594, A15 (2016)

[12] Motloch P., Hu W., in press, arXiv:1803.11526 (2018)

[13] Henning J.W., Sayre J.T., Reichardt C.L., et al., ApJ, 852, 97 (2018)

[14] Vikhlinin A., Burenin R.A., Ebeling H., et al., ApJ, 692, 1033 (2009)

[15] Vikhlinin A., Kravtsov A.V., Burenin R.A., et al., ApJ, 692, 1060 (2009)

[16] Mantz A.B., von der Linden A., Allen S.W., et al., MNRAS, 446, 2205 (2015)

[17] Rozo E., Wechsler R.H., Rykoff E.S., et al., ApJ, 708, 645 (2010)

[18] Sunyaev R.A. and Zeldovich Ya.B., Comments on Astrophysics and Space Physics, 4, 173 (1972)

[19] de Haan T., Benson B.A., Bleem L.E., et al., ApJ, 832, id.95 (2016)

[20] Planck Collaboration, Planck 2015 Results XXIV, A\&A, 594, A24 (2016)

[21] Burenin R.A. and Vikhlinin A.A., Astronomy Letters, 38, 347 (2012)

[22] Hoekstra H., Herbonnet R., Muzzin A., et al., MNRAS, 449, 685 (2015)

[23] Lyapin A. and Burenin R., Astronomy Letters, in press, (2018)

[24] Heymans C., Grocutt E., Heavens A., et al., MNRAS, 432, 2433 (2013)

[25] DES Collaboration, in press, arXiv:1708.01530 (2018)

[26] Tegmark M., Eisenstein D.J., Strauss M.A., et al., PRD, 74, 123507 (2006)

[27] Blake C., Kazin E.A., Beutler F., et al., MNRAS, 418, 1707 (2011)

[28] Parkinson D., Riemer-Soerensen S., Blake C., et al., MNRAS, 418, 1707 (2011)

[29] Alam S., Ata M., Bailey S., et al., MNRAS, 470, 2617 (2017)

[30] Burenin R.A., Astronomy Letters, 39, 357 (2013)

[31] Lesgourgues J. and Pastor S., Physics Reports, 429, 307, (2006) 